\title{
Transmission Performance of mm-Waves on Radio over Fiber Systems: Dispersion and Intermodulation Issues
}

\author{
Ricardo Avó, Paula Laurêncio, and Maria C.R. Madeiros \\ Center of Electronic, Optoelectronic and Telecommunications, Faculdade de Ciências e \\ Tecnologia, Universidade do Algarve, Campus de Gambelas, \\ 8005-139 Faro, Portugal \\ \{ricardoavo, plaurenc, cmedeiro\}@ualg.pt
}

\begin{abstract}
Next generation wireless networks must provide high broadband access, which can be achieved by combining the fiber optics and wireless technologies. In this paper we analyze a mm-wave radio over fiber (RoF) optical access network architecture, combining radio subcarrier multiplexing techniques to improve system efficiency with fiber dispersion mitigation provided by optical single sideband modulation techniques. Our results show the system degradation introduced by the fiber link, namely fiber dispersion and intermodulation effects.
\end{abstract}

Keywords: Radio over fiber, optical single sideband, subcarrier multiplexing, intermodulation distortion.

\section{Introduction}

Consumer bandwidth requirements are increasing, driven by applications requiring high speed data transmission such as regular file transfers which are becoming larger and an increasing number of transparent applications, such as IP Television (IPTV) and Voice over IP (VoIP). The consensus to overcome this bottleneck is to take optical fiber closer to the consumer. Additionally, these needs are placing increasing demands for more bandwidth allocation via wireless access networks. Nowadays, the most used technologies for Wireless Local Area Networks (WLAN) are 802.11b/g/a with throughputs up to $\sim 27 \mathrm{Mbps}$, the $802.11 \mathrm{n}$ should ease the problem with $\sim 100$ Mbps throughput [1]. However, such speeds do not match the wireline home networks provided by Fiber To The Home (FTTH) connections, such as $1 \mathrm{Gbit} / \mathrm{s}$ Internet. Other problematic scenarios are in-home IPTV distribution, especially with HDTV and future 3D HDTV, or even sharing content from a Personal Video Recorder (PVR) with different TV sets. The scenario on the Wireless Wide Area Networks (WWAN) connections is even worst, with theoretical maximum speeds of 14.4 Mbps with current High-Speed Packet Access (HSPA). The next generation technologies provide 42Mbps in HSPA+ and 100Mbps with Long Term Evolution (LTE). 
The increased bandwidth requirements cause spectral congestion at lower microwave frequencies, which are currently used in wireless access networks. In the millimeter-wave (mm-wave) region there is a large bandwidth available, with friendly regulation conditions, especially in the $60 \mathrm{GHz}$ band, which has at least $5 \mathrm{GHz}$ available bandwidth common thought out the main regions [2].

The reminder of this paper is organized as follows. In section 2 the contribution to the technological innovation is shown. In section 3, the network architecture is presented, emphasizing the effects of fiber dispersion and intermodulation introduced by the Optical Single Side Band (OSSB) modulator. Section 4 presents the simulation results for the downlink, and finally section 5 concludes the paper.

\section{Contribution to Technological Innovation}

Due to the small coverage area, $60 \mathrm{GHz}$ networks require a large number of base stations (BSs) to cover a service area. This requirement has led to the development of system architectures where functions such as signal, routing, processing, handover and frequency allocation are performed at the central office (CO). The best solution for connecting the $\mathrm{CO}$ with $\mathrm{BSs}$ in such radio network is via an optical fiber network, now known as radio over fiber (RoF) [3]. RoF has proven to be a viable solution to transport analog signals, e.g. CATV on hybrid fiber-coax networks or on PON networks with the RF overlay. By exploiting high bandwidth wavelength-division multiplexing (WDM) networks, an integrated efficient fiber radio backbone network can be realized, where mm-wave carriers are modulated with data and placed on a particular wavelength channel and delivered to a specific BS.

In this paper we analyze the downlink operation of a RoF network that delivers directly the mm-wave signals from the CO to the BSs. The transmission of mm-wave signals over optical fiber is highly impaired by the fiber chromatic dispersion. To minimize this effect OSSB is used. To further improve the system efficiency SubCarrier Multiplexing (SCM) is employed, this allows for the use of multiple independent radio channels in each BS. We consider using four independent SCM channels feeding each BS.

\section{System Description}

The downlink basic configuration is shown in figure 1. At the central office, four base-band $1 \mathrm{Gbit} / \mathrm{s}$ data signals modulate four different microwave carriers (58.1 $\mathrm{GHz}, 60.3 \mathrm{GHz}, 62.5 \mathrm{GHz}$ and $64.7 \mathrm{GHz}$ ) by using Binary Phase Shift Keying (BPSK) modulation. The modulated subcarriers are combined and the composite signal is used to externally modulate a laser using OSSB modulation. The optical signal is then delivered to a specific BS through an optical fiber link. At the BS, a photodiode with a bandwidth greater than the subcarrier frequency is used to directly detect the composite signal. The electrical mm-wave signal is filtered, amplified and directly delivered to the BS antenna. This approach has the advantage of a simplified BS design but it is susceptible to fiber chromatic dispersion that severely limits the transmission distance [4]. For this reason the architecture considered here employs OSSB modulation. 


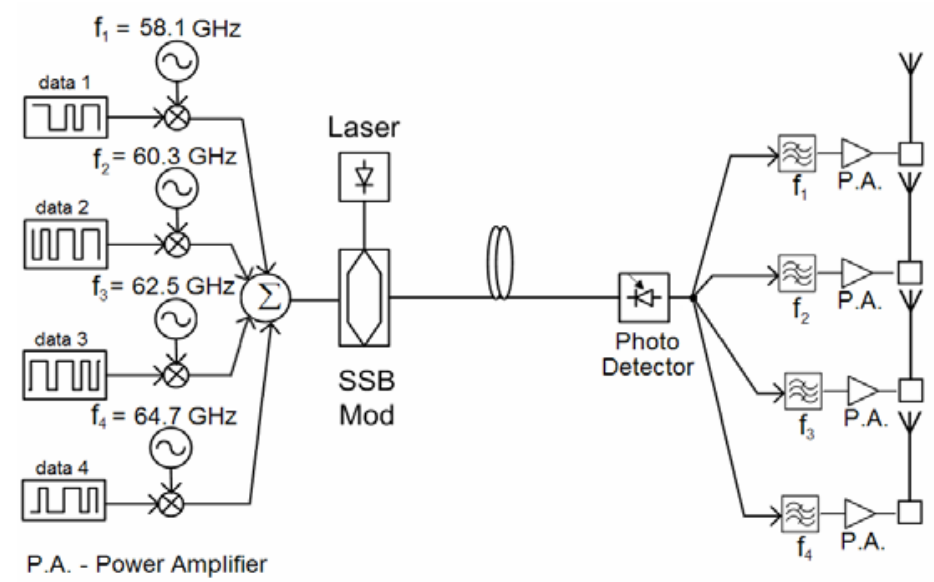

Fig. 1. Overall network architecture

The OSSB external modulator used in this work is the one proposed in [5]. The optical field at its output is given by:

$$
E_{M Z_{-} S S B}(t)=E_{i n}(t) \cos \left(x_{c} \pi m(t)-\pi / 4\right) \exp \left(j x_{c} \pi \hat{m}(t)\right) .
$$

where $E_{\text {in }}(t)=\sqrt{2 P_{o}} e^{j \omega_{c} t}$ is the optical field at the input of the MZ modulator, with optical angular frequency $\omega_{c}$ and average power $P_{o}, x_{c}$ is the channel modulation parameter, $m(t)$ represents the applied SCM electrical signal composed by $N$ channels using BPSK modulation and $\hat{m}(t)$ is its Hilbert transform. Assuming $m(t)$ to be a pure carrier at angular frequency $\omega_{m}$, the optical field at the output of the OSSB modulator can be written as a series of Bessel functions [6]:

$$
E_{M Z_{-} S S B}(t)=\sqrt{2 P_{0}} e^{j \omega_{c} t} \sum_{n=-\infty}^{\infty} \cos \left[(n-1) \frac{\pi}{4}\right] J_{n}\left(m_{I}\right) e^{j n\left(\omega_{m} t-\frac{\pi}{2}\right)} .
$$

where $J_{n}$ is n order Bessel function, the modulation index $m_{I}=\sqrt{2} x \pi A_{m}$ and $A_{m}$ is the $m(t)$ amplitude. For small values of $m_{I}$ only the fundamental harmonic is significant, under this circumstances the modulator can be considered linear and intermodulation introduced by the modulator is not an issue. However, for high values of $m_{I}$ other harmonics become relevant, as shown in figure $2 \mathrm{a}$ and may degrade the system performance, this is particularly important when combined with fiber dispersion.

\subsection{The Effect of Fiber Dispersion}

The ideal optical spectrum of a mm-wave over fiber is a signal which uses double sideband modulated (DSB), is composed by the optical carrier at optical frequency $f_{0}$ and by the mm-wave carrier $f_{i}$ located at the optical frequency $\pm f_{i}+f_{0}$. When the mmwave propagates through fiber modeled as a linear and dispersive link with a frequency response, $H_{L I N K}(z, \omega)=\exp \left(-j \beta_{2} \omega^{2} z / 2\right), \omega$ is the angular modulation 
frequency, $z$ is the longitudinal coordinate of the fiber, $\beta_{2}=-\lambda^{2} D /(2 \pi c)$ denotes the group velocity dispersion, with $D$ the dispersion parameter, $c$ the light speed in vacuum and $\lambda$ the carrier wavelength), the two optical field harmonics, $\pm f_{i}+f_{o}$, are affected by different phase changes due to the fiber dispersion. Furthermore, upon photodetection the two optical field harmonics interact in such a way leading to a detected photocurrent that change with distance and frequency, as shown in figure $2 b$.

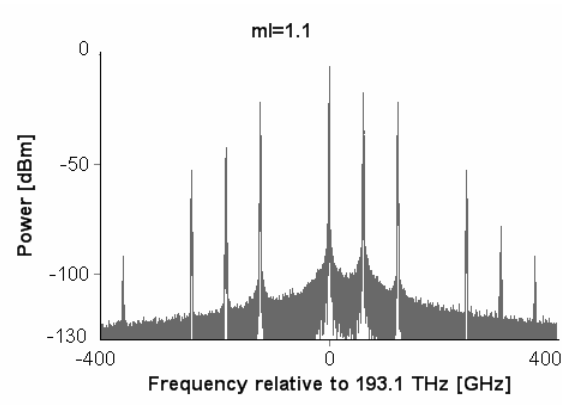

(a)

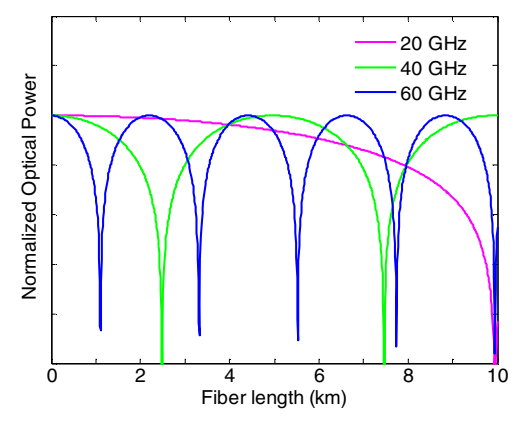

(b)

Fig. 2. a) Optical spectrum at the output of the OSSB modulator, for $m_{I}=1.1$. b) Normalized received optical power for an ideal optical system employing ODSB. Three RF carrier frequencies are considered, $20 \mathrm{GHz}, 40 \mathrm{GHz}$ and $60 \mathrm{GHz}$.

Theoretically, if optical single sideband modulation is employed, and the modulation index is small, dispersion does not affect the propagation of the mm-wave signal [6].

Note that, in practical systems the OSSB modulator, owing to its inherent nonlinearity, specially for higher values of $m_{I}$, generates other significant harmonic components as illustrated in figure $2 \mathrm{a}$, and therefore the system behaviour approaches an Optical Double Side Band (ODSB).

\subsection{The Effect of Intermodulation Distortion}

For an OSSB/SCM system, intermodulation distortion mainly arises from the nonlinear characteristics of the optical modulator, the linear fiber dispersion and from the square law characteristics of the photodetection process. Here, we focus on the intermodulation distortion arising from the nonlinear characteristics of the optical modulator. Assuming $m(t)$ to represent the applied SCM electrical signal composed by $\mathrm{N}$ channels and $\hat{m}(t)$ is its Hilbert transform:

$$
m(t)=A_{m} \sum_{i=1}^{N} u_{i}(t) \sin \left(\omega_{i} t+\theta_{i}\right) \quad \hat{m}(t)=A_{m} \sum_{i=1}^{N}-u_{i}(t) \cos \left(\omega_{i} t+\theta_{i}\right)
$$

where $\omega_{i}$ represents the RF angular subcarrier of the $i_{t h}$ channel $u_{i}(t)= \pm 1$ for BPSK (+ for ' 1 ' and - for ' 0 '), and $\theta_{i}$ is the subcarrier phase. 
The optical field at the output of the OSSB modulator, $E_{M Z_{-} S S B}(t)$, can be expanded as a series of Bessel functions, for small channels modulation indexes (1) and can be rewritten as:

$$
\begin{aligned}
E_{M Z_{-} S S B}(t) & =\sqrt{P_{o} / 2} \exp [-j \pi / 4]\left\{\prod_{i=1}^{N}\left\{1-j \frac{\sqrt{2} m_{I}}{2}\left[-s_{i}(t)-\hat{s}_{i}(t)\right]\right\}\right. \\
& \left.+\mathrm{j} \prod_{i=1}^{N}\left\{1-j \frac{\sqrt{2} m_{I}}{2}\left[s_{i}(t)-\hat{s}_{i}(t)\right]\right\}\right\} \exp \left(j \omega_{c} t\right)
\end{aligned}
$$

with channel modulation index $m_{I}=\sqrt{2} x_{c} \pi A_{m}$.

The optical field at the output of a fiber link of length $z$ is given by [7],

$$
\begin{gathered}
E_{\text {out }}(t)=\sqrt{P_{o} / 2} \exp (-j \pi / 4) \times\left\{\prod_{i=1}^{N}\left\{1-j \frac{\sqrt{2} m_{I}}{2}\left[-s_{i}(t)-\hat{s}_{i}(t)\right] \exp \left(-j \frac{\beta_{2}}{2} i^{2} \omega^{2} z\right)\right\}\right. \\
\left.+j \prod_{i=1}^{N}\left\{1-j \frac{\sqrt{2} m_{I}}{2}\left[s_{i}(t)-\hat{s}_{i}(t)\right] \exp \left(-j \frac{\beta_{2}}{2} i^{2} \omega^{2} z\right)\right\}\right\} \times \exp \left(j \omega_{c} t\right)
\end{gathered}
$$

Furthermore, as the envelope of the optical field is detected by a photodetector with responsivity, $R_{\lambda}$, the detected photocurrent is proportional to the envelope of optical field at the fiber output. Considering the intermodulation products, the temporal expression of the detected current may be expressed as $i(t) \approx i_{D C}+i_{\text {sig }}(t)+i_{\text {int }}(t)$, where $i_{D C}$ is the detected current DC term, $i_{\text {sig }}(t)$ is the SCM electrical signal, $i_{\text {int }}(t)$ accounts for the intermodulation products, which acts as noise.

In such systems it is important to consider the intermodulation effects between the sub-carriers as well as between sub-carrier and higher harmonics when high modulation indexes are considered.

For small modulation indexes intermodulation can be calculated using (5), however, for high values of $m_{I}$ system simulation is necessary. When using system simulation the bandwidth of each channel and not just the carrier are taken into account.

\section{Simulation Results}

System simulations were performed using VPI TransmissionMaker ${ }^{\mathrm{TM}}$. The library blocks of the simulator were complemented when necessary with blocks implemented using MathWorks software tool MATLAB®. The light source considered in the simulation is an ideal laser, with $1 \mathrm{~mW}$ of emission power.

Figure 3 shows the simulation overview showing the signal at some system points. The $1 \mathrm{Gbit} / \mathrm{s}$ data which is filtered by a $100 \%$ roll over square root raised cosine filter. The filtered data modulates the mm-wave carrier using BPSK modulation. The 4 channels are mixed and used to modulate the optical carrier to OSSB with a $m_{I}=0.2$.

After transmission through standard singlemode fiber, operating at $1553 \mathrm{~nm}, 16$ $\mathrm{ps} /(\mathrm{nm} . \mathrm{km})$ dispersion coefficient, the signal is directly detected by a high speed PIN photodetector. The receiver considered introduces a noise current of $10 \mathrm{e}^{-12} \mathrm{~A} / \sqrt{\mathrm{Hz}}$. The photodetected channels are separated by mixing with the respective carrier and filtered by a $100 \%$ roll over square root raised cosine filter. 


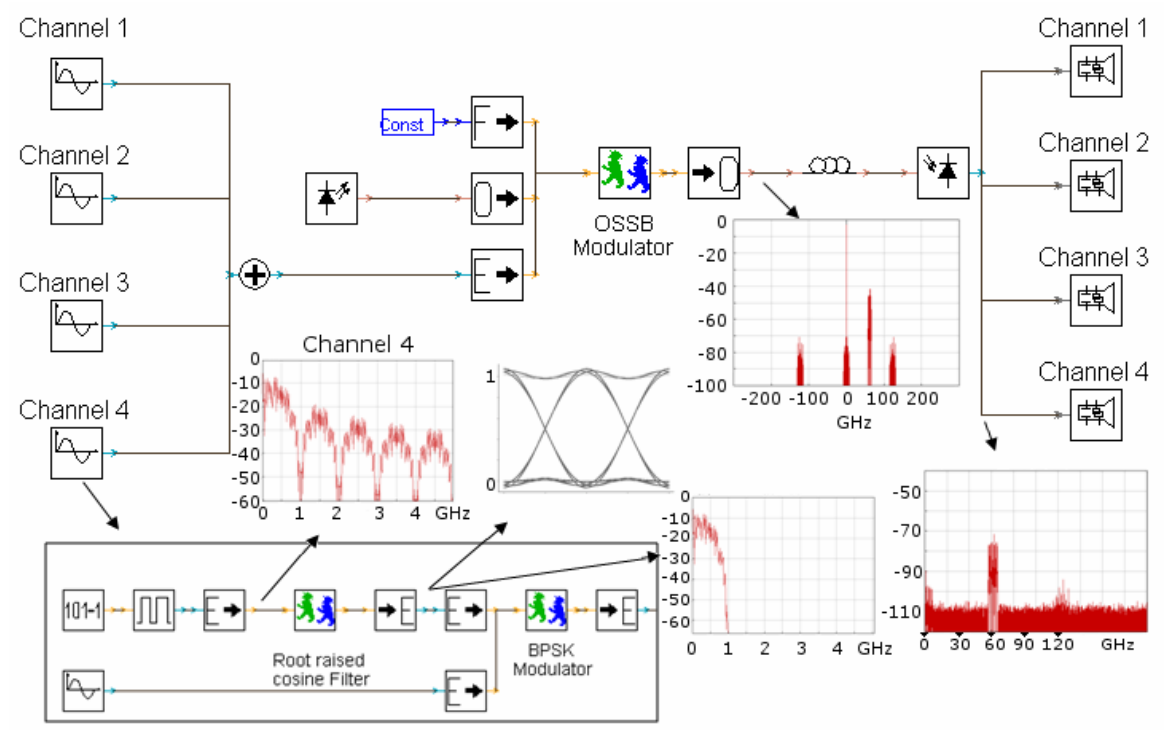

Fig. 3. Overall simulated system with illustrative signals

To measure the intermodulation distortion falling in a given channel, the channel under analysis is switched off, and the intermodulation power falling in the channel is measured. Figure $4 \mathrm{a}$ and $4 \mathrm{~b}$ show the simulated received electrical spectrum when the $60 \mathrm{GHz}$ channel is switched off, for two modulation indexes $m_{I}=0.44$ and $m_{I}=1.1$ respectively and a fiber length of $5 \mathrm{~km}$. For $m_{I}=0.44$ the intermodulation power falling in the $60 \mathrm{GHz}$ channel is not relevant whereas for $m_{I}=1.1$, the intermodulation power resulting from the sum of the different optical field harmonic beats is significant. Intermodulation power also depends on fiber length, since due to dispersion the different optical field harmonics suffer phase changes which depend on fiber length leading to total intermodulation power variations. In some situations, the intermodulation power falling in a given channel can even decrease with distance, as shown in figure $4 \mathrm{c}$.
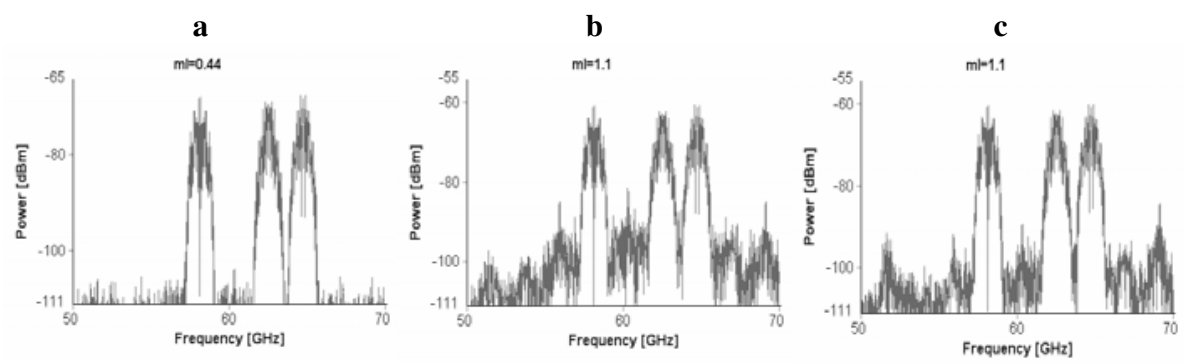

Fig. 4. Received electrical spectrum, showing intermodulation power falling on the $60 \mathrm{GHz}$ channel. a) $\mathrm{m}_{\mathrm{I}}=0.44$ fiber length of $5 \mathrm{~km}$. b) $\mathrm{m}_{\mathrm{I}}=1.1$ fiber length of $5 \mathrm{~km}$. c) $\mathrm{m}_{\mathrm{I}}=1.1$ fiber length of $50 \mathrm{~km}$. 

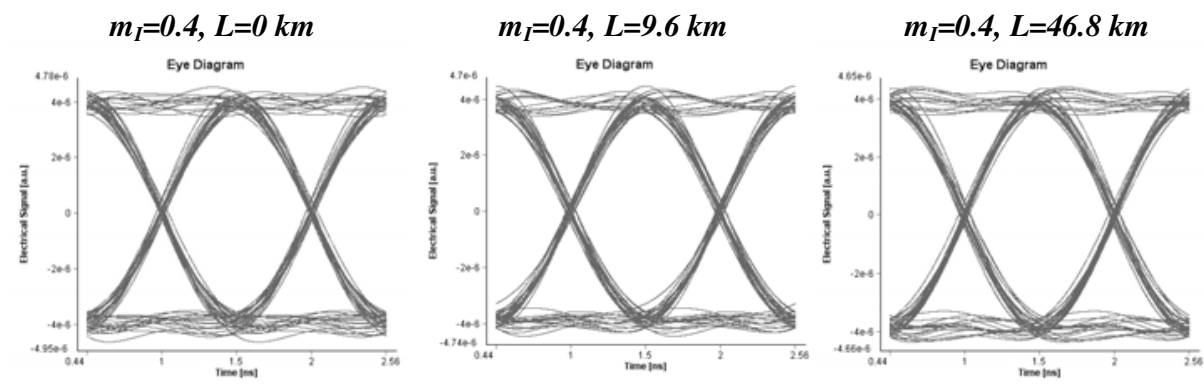

$m_{I}=1.1, L=0 \mathrm{~km}$
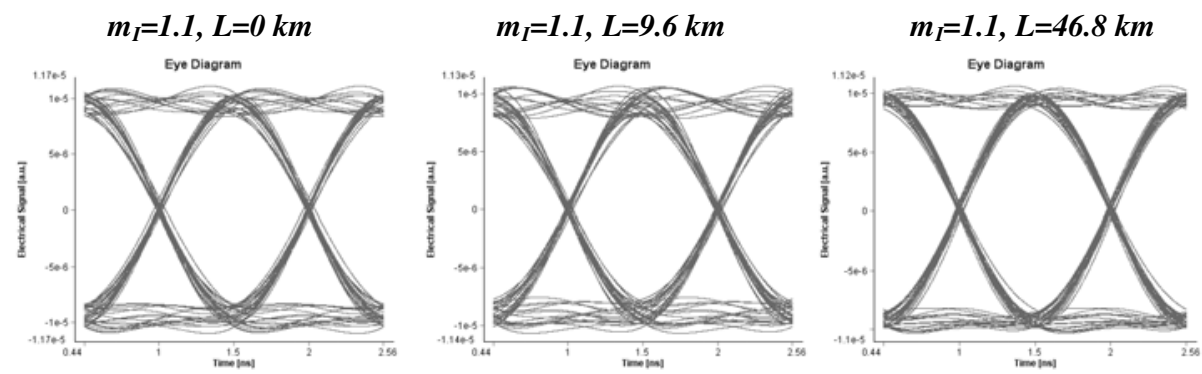

Fig. 5. Received eye diagrams for the $60 \mathrm{GHz}$ channel considering different values of modulation index $\mathrm{m}_{\mathrm{I}}$ and fiber lengths $\mathrm{L}$

Figure 5 shows the eye diagrams of the received data of the $60 \mathrm{GHz}$ channel corresponding to the two modulation indexes and different fiber lengths. For a fiber length of $0 \mathrm{~km}$ as expected for $m_{I}=1.1$, the received eye presents higher distortion when compared with $m_{I}=0.4$. For small values of $\mathrm{m}_{\mathrm{I}}$ intermodulation is not relevant and the system performance is not significantly affected by dispersion, therefore the received eye diagrams are kept widely open when transmission distance increases. However, the most interesting results arise when the distortion introduced by the OSSB modulator is combined with fiber dispersion. For high modulation indexes $m_{I}=1.1$, the combined effect of dispersion and intermodulation give rise to oscillations on the system performance, for example for a fiber length of $46.8 \mathrm{~km}$ the eye is wider than for $9.6 \mathrm{~km}$. Although not shown in this paper, we note that these results depend strongly on the mm-wave carrier frequency.

\section{Conclusions}

Although SSB improves the system reach, the intermodulation distortion and fiber dispersion are still the main issues, as shown by our numerical simulation. This implies a careful consideration when choosing the network architecture.

Other optical modulation techniques should be studied, such as optical carrier suppression or optical filtering, to compare the fiber chromatic dispersion effect with our OSSB system. The data transmission rate should be analyzed and its robustness should be compared to different modulation formats. Orthogonal Frequency Multiplexing (OFDM) could be a good candidate to improve the system performance 
when using high bit rates by dividing the transmission channel in several low rate channels.

Acknowledgments. This work was supported by the Portuguese Foundation for Science and Technology within the project PTDC/EEA-TEL/71678/2006, Research Unity, I\&D 631 and by the PhD grant SFRH/BD/29138/2006.

\section{References}

1. Chang, G., Yu, J., Jia, Z.: Architectures and Enabling Technologies for Super-Broadband Radio-over-Fiber Optical-Wireless Access Networks. In: 2007 IEEE International Topical Meeting on Microwave Photonics, pp. 24-28 (2007)

2. Ohata, K., Maruhashi, et al.: 1.25Gbps Wireless Gigabit Ethernet Link at $60 \mathrm{GHz}-\mathrm{Band}$. In: 2003 IEEE MTT-S International Microwave Symposium Digest, vol. 1, pp. 373-376 (2003)

3. O'Reilly, J.J., Lane, P.M., Attard, J., Griffin, R.: Broadband wireless systems and networks: an enabling role for radio-over-fibre. Philos. Trans. R. Soc. London 358, 2297-2308 (2000)

4. Schmuck, H.: Comparison of optical millimeter-wave system concepts with regard to chromatic dispersion. Electron. Lett. 31, 1848-1849 (1995)

5. Sieben, M., Conradi, J., Dodds, D.E.: Optical single sideband transmission at $10 \mathrm{~Gb} / \mathrm{s}$ using only electrical dispersion compensation. J. Lightwave Technol. 17, 1742-1749 (1999)

6. Laurêncio, P., Medeiros, M.C.R.: Dynamic range of optical links employing optical single side-band modulation. IEEE Photonics Technol. Lett. 15, 748-750 (2003)

7. Laurêncio, P., Simões, S., Medeiros, M.C.R.: Impact of the combined effect of RIN and intermodulation distortion on OSSB/SCM systems. J. Lightwave Technol. 24, 4250-4260 (2006) 